\title{
High-quality 3D shape measurement using saturated fringe patterns
}

\author{
Bo Chen ${ }^{\mathrm{a}}$ and Song Zhang $\mathrm{b}, *$

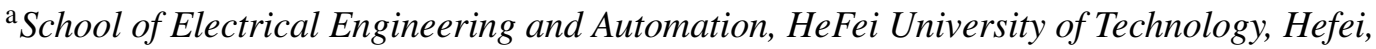 \\ China 230009 \\ ${ }^{\mathrm{b}}$ School of Mechanical Engineering, Purdue University, West Lafayette, IN 47907
}

\begin{abstract}
This paper proposes a method to potentially conquer one of the challenges in the optical metrology community: optically measuring three-dimensional (3D) objects with high surface contrast. We discover that for digitally equally phase-shifted fringe patterns, if the fringe period $P$ is an even number, the $N=P / 2 \times k,(k=1,2,3, \ldots)$ step algorithm can accurately recover phase even if the fringe patterns are saturated; and if $P$ is an odd number, $N=P \times k$ step algorithm can also accurately recover phase even if the fringe patterns are saturated. This finding leads to a novel method to optically measure shiny surfaces, where the saturation due to surface shininess could be substantially alleviated. Both simulations and experiments successfully verified the proposed method.
\end{abstract}

Key words: Fringe projection, fringe analysis, High dynamic range, 3D shape measurement.

\section{Introduction}

Optically measuring a high-contrast 3D surface (e.g., shiny surface) poses significant challenges to the optical metrology community since it is difficult to obtain high-quality images for the whole surface with a single exposure.

One of the state-of-the-art methods for high-contrast 3D surface measurement is called high-dynamic range (HDR) 3D shape measurement. These HDR techniques can be classified into two categories: without pre-analysis $[1,2]$ and with pre-analysis

\footnotetext{
* Corresponding author. Tel.: 765496 0389; Fax: 765494 0539; Email: szhang15@purdue.edu
} 
$[3,4,5,6]$. The former blindly captures a sequence of images with different exposures and select the best quality exposure for each point, though successful, they often require some pre-knowledge about the object surface to ensure good quality measurements. The latter group captures a sequence of images with different exposures, analyzes these images to determines the optimal exposure for each point, and then measures the surface with optimal exposures. Although these methods could work well, they are typically very slow. In general, the HDR methods all require the acquisition of images with different exposures, posing challenges to apply them to high-speed applications where changing camera exposures quickly is usually not easy.

The second state-of-the-art methods developed to handle high-contrast surfaces is to adapt projected fringe patterns accordingly [7,8,9]. These methods essentially adjust projected fringe pattern contrast to ensure no saturation points on measured surfaces. They all work well assuming if the ambient light is close to be zero. However, the measurement accuracy could be compromised if the ambient light is strong since the SNR of the projected fringe pattern is substantially reduced.

Instead of changing camera exposures or projected fringe contrast, researchers also developed another method to handle high-contrast 3D surfaces by employing a special hardware called spatial light modulator [10]. This method uses a spatial light modulator in front of the camera to avoid saturated pixels by monitoring the input light intensity and thus adjust the transmittance of light to that pixel. However, such a modulation and sensing system could be quite expensive and complicated to be employed for applications where the cost is a major concern.

A special group of state-of-the-art methods developed to handle high-contrast surfaces either use polarizing filters $[11,12]$, or to perform measurements from different perspectives [13]. All these methods are developed mainly for shiny surface measurement. The former is effective to reduce spectacular effects, but substantially sacrifice light intensity due to polarization. The latter method reduces the shiny areas by measuring the surface from different angles. However, such a method increases system complexity, and post data processing burden since neither $3 \mathrm{D}$ data registration nor 3D data fusion is trivial for high-accuracy measurements.

Comparing with methods developed for high-contrast surface measurements and those methods developed for shiny surface measurements, one may notice that the former requires change exposures of a camera or adapt the contrast of the projected fringe patterns, or varies the transmittance of the special hardware, while the latter does not. Apparently the former is less likely than the latter to perform real time measurements. The goal of all these methods is to capture high-quality images for further analysis (i.e. with high signal to noise ratio, SNR) since the camera only has limited dynamic range. The underlying assumption is that if the camera is saturated, no good measurements could be achieved. 
This paper argues that the saturated camera images can be used to perform highquality 3D shape measurement for digital fringe projection (DFP) methods provided that 1) proper phase-shifting algorithms are used, 2) fringe patterns are generated discretely, and 3) there is no phase-shift error. Both simulation and experimental results will be presented to validate the proposed idea.

Section 2 discusses the principle behind N-step phase-shifting algorithm. Section 3 shows simulation results. Section 4 presents experimental validation, and Sec. 6 summarizes this paper.

\section{Principle}

Phase-shifting-based algorithms are extensively used in practical 3D shape measurement systems due to their speed, accuracy, resolutions, and other merits over other methods. In general, $\mathrm{N}$ equally phase-shifted fringe pattern can be mathematically described as

$$
I_{k}(x, y)=I^{\prime}(x, y)+I^{\prime \prime}(x, y) \cos (\phi+2 k \pi / N),
$$

where $I^{\prime}(x, y)$ represents the average intensity, $I^{\prime \prime}(x, y)$ the intensity modulation, and $\phi(x, y)$ the phase to be solved for. Simultaneously solving these $N$ equations leads to

$$
\phi(x, y)=-\tan ^{-1}\left[\frac{\sum_{k=1}^{N} I_{k} \sin (2 k \pi / N)}{\sum_{k=1}^{N} I_{k} \cos (2 k \pi / N)}\right] .
$$

This equation produces wrapped phase whose value ranges $[-\pi,+\pi)$. A spatial or temporal phase-unwrapping algorithm can be adopted to remove $2 \pi$ discontinuities and obtain a continuous phase map. The continuous phase can then be used to reconstruct $(x, y, z)$ coordinates.

Typically, close-to-ideal sinusoidal patterns and accurate phase shifts are required to obtain accurate phase, and thus the community has been striving to produce as high-quality sinusoidal patterns as possible, and as accurate phase shifts as possible. In a DFP system, phase shifts can be accurately generated due to digital fringe generation nature, and thus the sinusoidality is the major concern. As a result, fringe pattern saturation should always be avoided because saturation means the use of non-sinusoidal fringe patterns. Therefore, it is a common belief that it is difficult to accurately measure high-contrast surfaces (e.g., shiny surface) at once because it is difficult to obtain high-quality sinusoidal patterns across the whole surface with a single exposure. As such, it drives the development HDR and shiny surface measurement techniques in the community, as discussed in Sec. 1.

This paper argues that the saturated patterns can be used to recover accurate phase and thus to perform high-accuracy $3 \mathrm{D}$ shape measurement provided that 1 ) a proper 
phase-shifting algorithm is used, 2) all fringe patterns are generated discretely, and 3) there is no phase-shift error. For a DFP system, 2) and 3) can be easily ensured and thus the only major issue is the use of a phase-shifting algorithm. We discover that for equally phase-shifted fringe patterns, if the fringe period $P$ is an even number, $N=P / 2 \times k(k=1,2, \ldots)$ step algorithm can accurately recover phase even if fringe patterns are saturated; and if $P$ is an odd number, $N=P \times k$ step algorithm can also accurately recover phase even if fringe patterns are saturated. It should be noted that $k=1$ is sufficient to eliminate saturation error, yet random phase noise can be reduced by using $k>1$.

The next section illustrates the proposed idea through simulation, and Sec. 4 experimentally verifies the success of the proposed method.

\section{Simulation}

We first simulated the effects of saturation with different amount of saturation by a scale factor $S$. The ideal 8-bit fringe patterns with for a N-step phase-shifting algorithm with equal phase shifts can be described as

$$
I_{k}(i, j)=S \times 127.5[1+\cos (2 j \pi / P+2 k \pi / N)],
$$

where $P$ is the fringe period in pixels. Assume an 8-bit camera is used, if $S>1$, the fringe patterns are saturated and the resulting fringe patterns can be mathematically described as

$$
I_{k}^{S}(i, j)=\left\{\begin{array}{ll}
I_{k}(i, j) & I_{k}(i, j) \leq 255 \\
255 & \text { otherwise }
\end{array} .\right.
$$

Figure 1 shows examples when the scale factor $S$ varies from 1.0 to 6.0 and fringe period $P=40$ pixels. Figures 1 (a) and 1(d) shows the result when $S=1$, ideal sinusoidal patterns are generated. When $S$ increases, the saturation area becomes larger and larger; and when $S=6$, the patterns are substantially saturated, making the majority areas being saturated, as shown in Fig. 1(c) and Fig. 1(f).

We computed the phase error for all these patterns when using the $N=P / 2$ step phase-shifting algorithm, the phase root-mean-square error (rmse) is $4.53 \times 10^{-4}, 2.08 \times$ $10^{-15}, 2.06 \times 10^{-15} \mathrm{rad}$ for $S=1.0,1.5$ and 6.0, respectively. Clearly, all these numbers are very small. One may notice that using ideal sinusoidal patterns actually produces larger error than the saturated patterns, which might be a result of quantization effect.

We then simulated different levels of saturation using the $N=P / 2$ step phaseshifting algorithm for fringe period of $P=40$ pixels. Figure 2 shows the results. Again, it shows that when $1.0 \leq S \leq 7.5$, all phase errors are all negligibly small, 


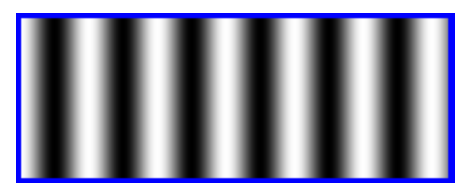

(a)

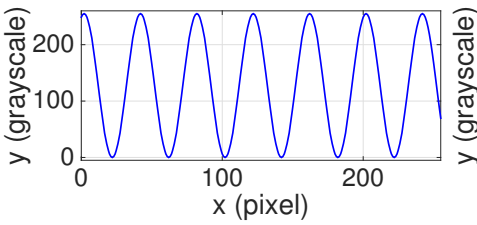

(d)

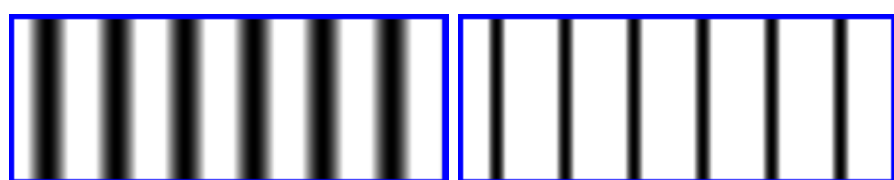

(b)

(c)

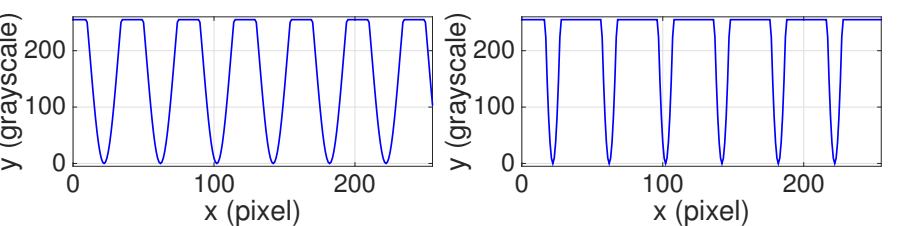

(e)

(f)

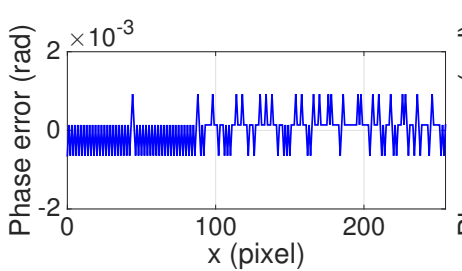

(g)

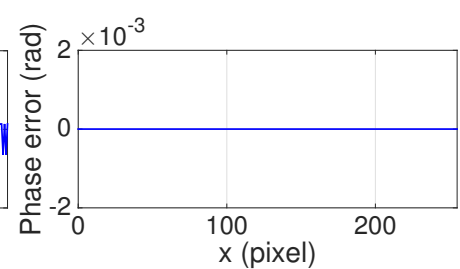

(h)

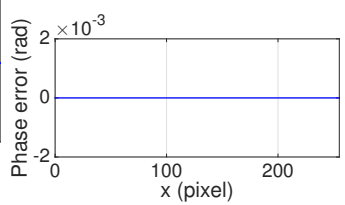

(i)

Fig. 1. Example fringe patterns with different level of saturation (fringe period $P=40$ pixels, and step $N=20$ ). (a)-(c) Shows one of fringe patterns with scale factor of 1.0, 1.5, 6.0, respectively; (d)-(f) respective shows cross section of the above fringe pattern; (g)-(i) respectively shows the phase error plot using $N=P / 2$ step phase-shifting algorithm, and the phase rms error is $4.53 \times 10^{-4}, 2.08 \times 10^{-15}, 2.06 \times 10^{-15}$ rad respectively.

demonstrating that fringe saturation indeed will not jeopardize phase quality if $N=$ $P / 2$ equally phase-shifted fringe patterns are used, and the fringe period is an even number.

All previous simulations did not include noise effect, and thus are difficult to be realized in practice. To emulate practical implementations, we added Gaussian noise $(10 \mathrm{~dB})$ to the signal and then analyzed phase rms error again. Figure 2(b) shows the corresponding result with noise. Comparing with the noise-free systems, the systems with added noise 1) have larger phase rms errors (as expected); and 2) increases phase errors when saturation becomes too much. Nevertheless, the phase rms error are at similar or even lower level when the fringe patterns are saturated vs the non-saturated fringe patterns.

We also simulated different breadths (i.e., fringe period $P$ ) of fringe patterns with saturated fringe patterns when $S=3.0$, Figures 3(a) and 3(c) show the results when the fringe period ranges from 6 to 60 pixels (note here that only even number of fringe periods are used). Once again, the resultant phase errors are all negligible when $N=P / 2$ step phase-shifting algorithm is used. This figure further shows that if $N=P$ step phase-shifting algorithm is used, the resultant phase errors are also at a similar level as those non-saturated patterns. 


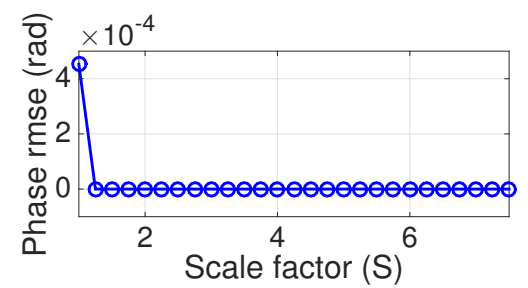

(a)

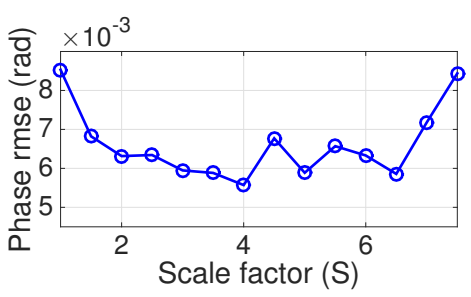

(b)

Fig. 2. Phase rms error with different level of saturation (fringe period $P=40$ pixels, and step $\mathrm{N}=20$ ). (a) Noise free signals; (b) with added Gaussian noise to the signals.

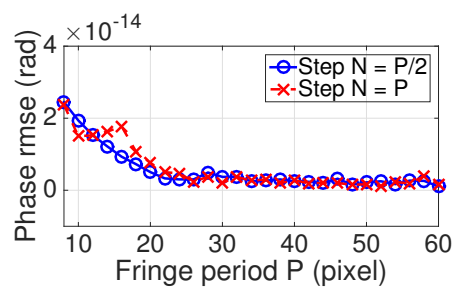

(a)

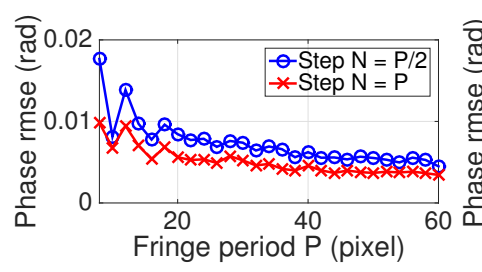

(c)

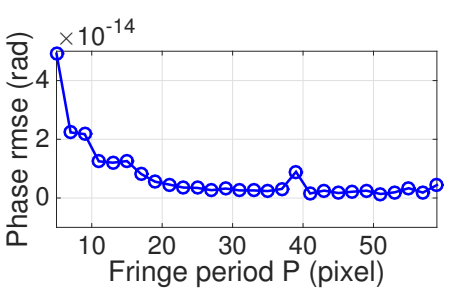

(b)

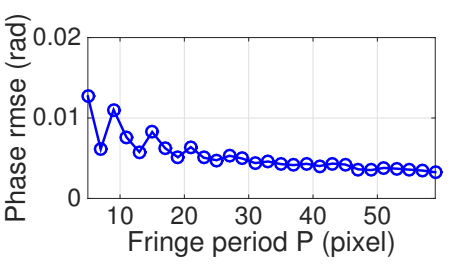

(d)

Fig. 3. Phase rms error with different fringe period $P$ (scale $\mathrm{S}=3.0$ ). (a) Even number $P$ and noise-free signals; (b) odd number $P$ and noise-free signals; (c) Even number $P$ and added Gaussian noise signals; (d) odd number $P$ and added Gaussian noise signals.

Furthermore, we simulated the case when $N=P$ step phase-shifting algorithm is applied to odd number fringe periods (i.e., $P$ is an odd number). Figures 3 (b) and 3(d) show the results as fringe period varies from 5 to 59 and $S=3.0$. Once again, the phase can be accurately recovered from the saturated patterns.

\section{Experimental Results}

We developed a 3D shape measurement system to evaluate the proposed algorithm. The test system uses a digital-light-processing (DLP) projector (Model: Dell M115HD) with a resolution of $1280 \times 800$. A digital CCD camera (Model: the Imaging Source DMK 23UX174) with a resolution of $1280 \times 1024$ is used to capture the scenes. The camera is attached with a $25 \mathrm{~mm}$ focal length lens (Model: Fujinon HF25SA-1). In this research, we used the N-step single-frequency fringe patterns to obtain the wrapped phase map, and a temporal phase unwrapping algorithm to obtain continuous unwrapped phase that was further converted to $(x, y, z)$ 
using the calibrated system. The temporal phase unwrapping method employed in this research was to use 7 additional gray-coded binary patterns to determine fringe order, and to adopt a computational framework discussed in Ref. [14] to remove unwrapping artifacts. Overall the minimum number of total images required to retrieve accurate wrapped phase without saturation error is $P / 2$ if $P$ is an even number, or $P$ if $P$ is an odd number. In this research, we used 7 additional images for temporal phase unwrapping, and bring the overall image number required for one 3D shape recovery to be $P / 2+7$ ( $P$ is an even number) or $P+7$ ( $P$ is an odd order). For example, if $P=20$ pixels, we can chose $N=10$, and the total number of images required to recover one 3D shape is 17 . It should be noted that using 7 images for temporal phase unwrapping may not be optimal since there are methods $[15,16]$ developed that only require one additional image for temporal phase unwrapping, or methods $[17,18,19,20]$ that do not require any additional image acquisition but rather use one more camera. The system was calibrated using the simple reference-plane-based approach as described in Ref. [21].

We first measured a flat board with three different levels of saturation by changing camera exposure time. Figure 4 shows the results of three representative exposures (Exposure 2, 3 and 5) out of 9 different exposures. In these experiments, we used fringe period of $P=40$ pixels and 20 (i.e., $N=P / 2$ ) equally phase-shifted fringe patterns for phase retrieval. Figures 4(a)-4(c) show the cross sections of the phaseshifted fringe patterns, and Figures 4(d)-4(f) show the corresponding phase error. The phase error is determined by taking the difference between an ideal phase and the recovered phase. Phase rms error for Fig. 4(d) is 0.0051 rad, Fig. 4(e) is 0.0047 rad, Fig. 4(f) is $0.0055 \mathrm{rad}$. They are all very small even when the patterns are substantially saturated, as shown in Fig. 4(c).

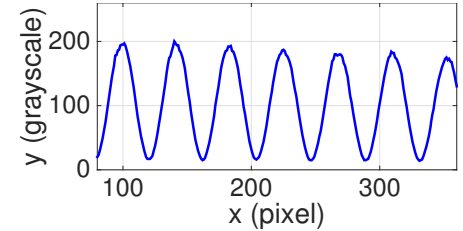

(a)

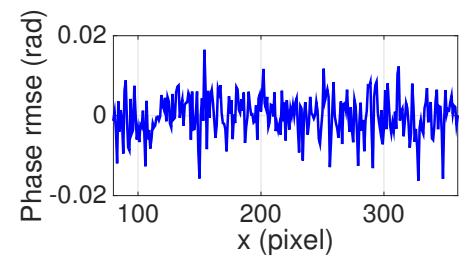

(d)

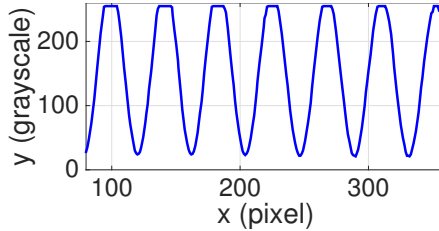

(b)

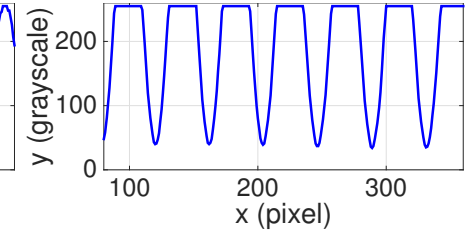

(c)

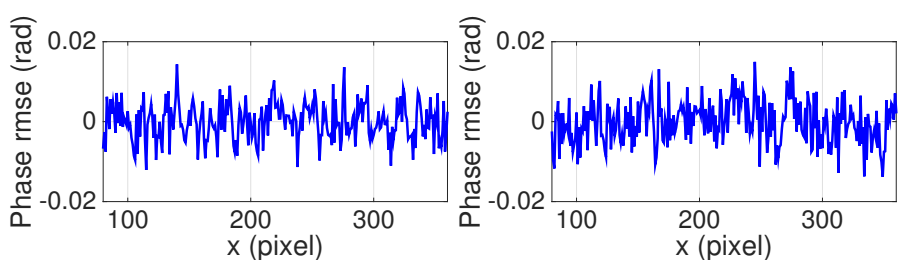

(e)

(f)

Fig. 4. Measurements of a flat surface with different amount of saturation (fringe period $P=40$ pixels, and step $N=20$ ). (a)-(c) Cross section of one of the phase-shifted fringe patterns; (d)-(f) corresponding phase error plot for (a), (b), and (c), and the rms error is respectively $0.051 \mathrm{rad}, 0.0047 \mathrm{rad}$, and $0.0055 \mathrm{rad}$.

Figure 5(a) shows the phase rms error for 9 different exposures when the fringe 
period $P=40$ pixels and $N=20$; Figure 5(b) shows the phase rms error for different number fringe periods and different phase shifting algorithms with a fixed exposure level. Clearly, all these errors are all consistently small, confirming our simulation results shown in Fig. 2(b) and Fig. 3(c)-3(d).

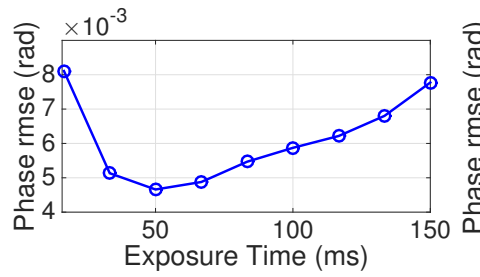

(a)

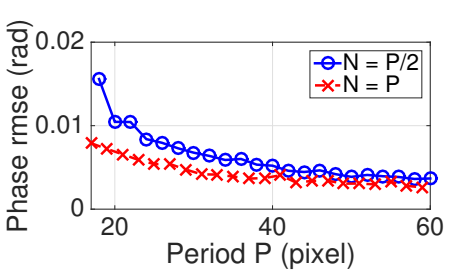

(b)

Fig. 5. Phase rms error with different fringe period and different exposures. (a) Phase rms error with different level of saturation (fringe period $P=40$ pixels, and step $N=20$ ); (b) phase rms error with different fringe period $P$.

We then measured a complex statue with different levels of saturation by changing camera exposure time. Video 1 (corresponding snapshot image shown in Fig. 6) shows all 12 different exposure times: $1 / 120 \times m$ sec with $m=1,2, \ldots, 12$. On the video, the left shows one of the 18-phase-shifted fringe patterns, and the right shows the recovered 3D shape. Figure 7 shows four representative frames with different exposure times from no saturation to substantial saturation. For all these experiments, we used fringe period $P=36$ pixels and phase-shifting step number $N=18$. Figure 7(a)-7(d) respectively shows one of the nine phase-shifted fringe patterns at 4 different exposures, respectively; and Figures 7(e)-7(h) show the corresponding 3D reconstructed shapes. These figures as well as Video 1 show that the differences between using saturated and non-saturated fringe patterns are very minor, confirming that the proposed algorithm can work well for complex diffuse surfaces.

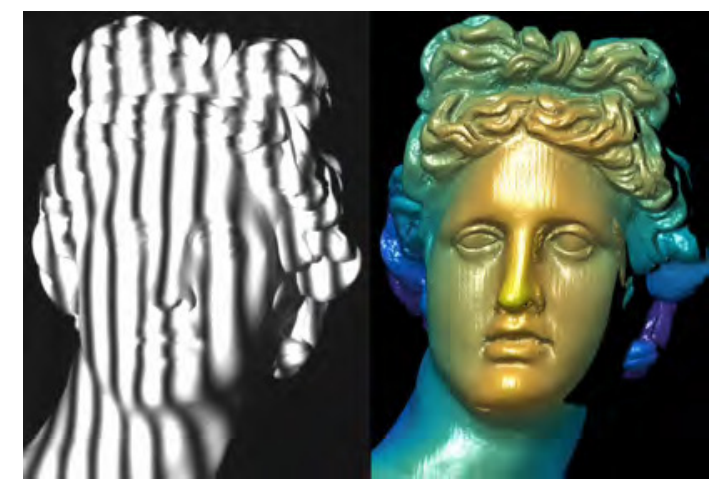

(a)

Fig. 6. Supplemental video and the video can be downloaded from https://engineering.purdue.edu/ZhangLab/videos/OLE_video1.mp4.

Finally, we measured a shiny surface including a metal part, PCB board, and different shapes to verify that the proposed method can actually be used to measure 


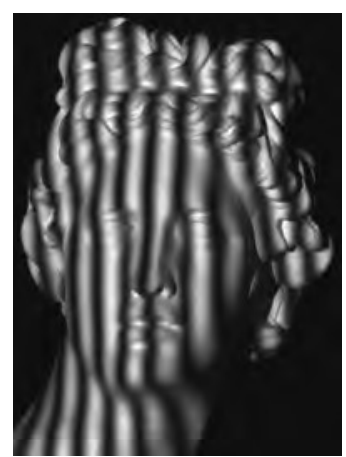

(a)

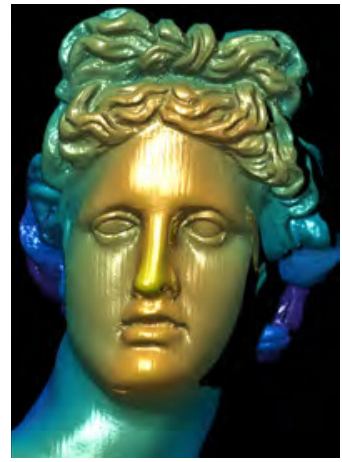

(e)

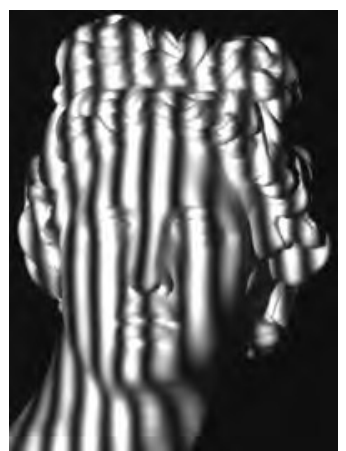

(b)

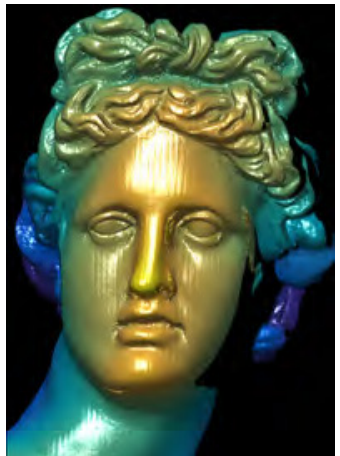

(f)

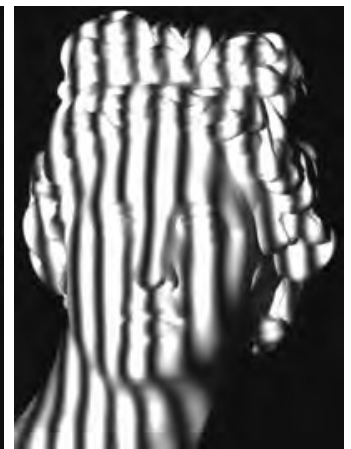

(c)

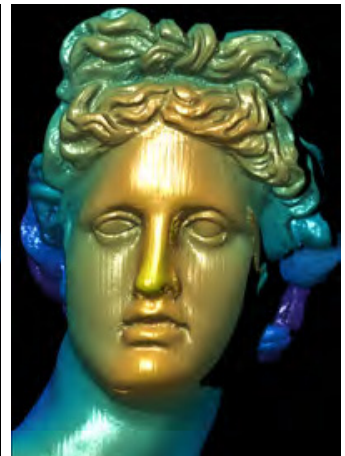

$(\mathrm{g})$

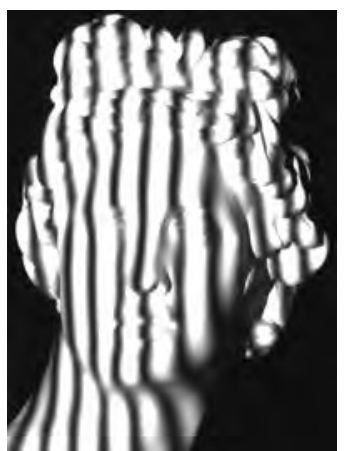

(d)

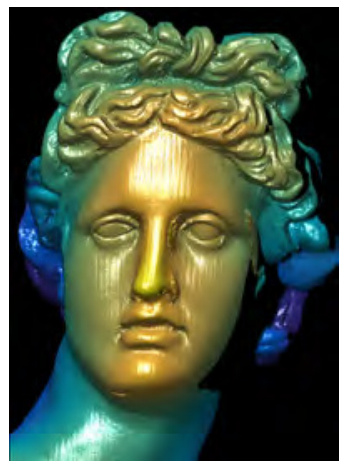

(h)

Fig. 7. Measurements of a complex statue with different amount of saturation (fringe period $P=36$ pixels, and step $N=18$ ); Associated Video 1. (a)-(d) One of the phase-shifted fringe patterns for each exposure; (e)-(h) corresponding 3D reconstructions for the exposure used to capture fringe patterns shown in (a), (b), (c) and (d), respectively.

shiny surfaces. Figure 8 show measurement results. Figure $8(a)$ shows the photograph of the part. Figure 8(b) shows one of the nine phase-shifted fringe patterns where some areas are saturated. Figure 8(c) shows the recovered 3D shape. Clearly, although the surface is shiny, causing fringe patterns to be partially statured, the whole surface is still satisfactorily measured. This experiment successfully verified that the proposed algorithm can be used to measure shiny objects.

\section{Discussion}

Comparing with the state-of-art methods to measure high-contrast 3D surfaces discussed in Sec. 1, the proposed method does not require any changes to the measurement conditions (e.g. camera exposure, projection fringe contrast), does not need any special hardware (e.g., polarization filter, spatial light modulator), or preknowledge of the measured object. The only requirement is to capture sufficient number of fringe patterns for a given fringe periods. 


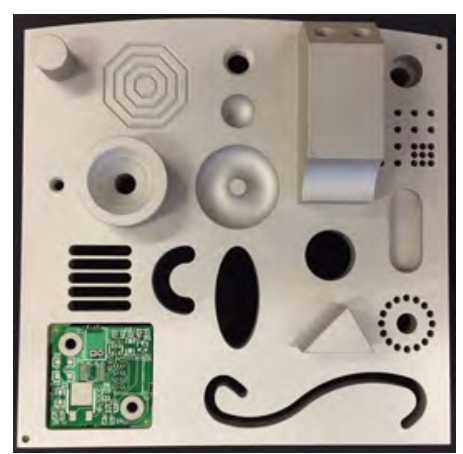

(a)

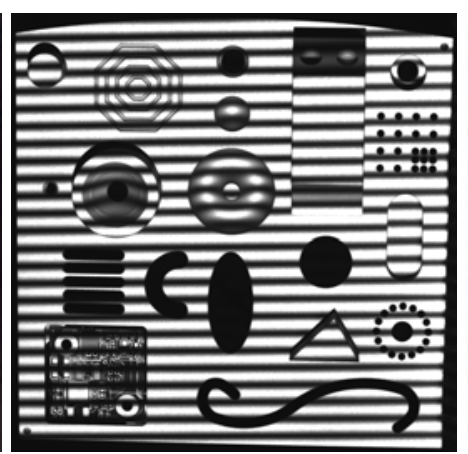

(b)

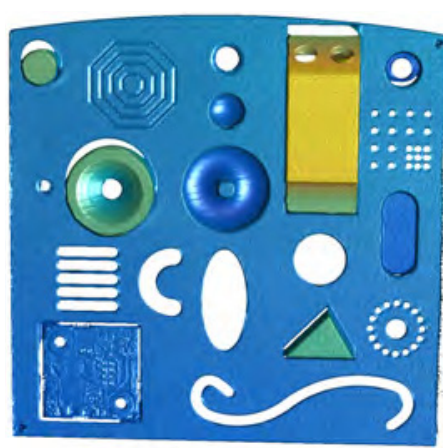

(c)

Fig. 8. Experimental results of a shiny object (fringe period $P=18$ pixels, and step $N=9$ ). (a) Photograph of the object; (b) one of the phase-shifted fringe patterns; (c) 3D reconstruction.

The idea of using more phase-shifted fringe patterns has been attempted before. Chen et al. [22] and $\mathrm{Hu}$ et al. [23] proposed to capture more phase-shifted fringe patterns and select at least three unsaturated fringe patterns for phase recovery. The underlying assumption of this method is still that saturated fringe patterns cannot be used for high quality phase recovery, and thus 3D shape measurement. Jiang et al. [24] recently developed a real-time measurement system by only using three additional inverted fringe patterns. In Jiang's paper, it first brought the idea of directly using saturated fringe patterns to recover phase and demonstrated that even though less than three phase-shifted fringe patterns are not saturated at a given point, good quality phase (with small error) can be obtained. However, this paper, for the first time, proved that accurate phase can be recovered by using saturated fringe patterns.

\section{Conclusion}

This paper has presented our recent finding on achieving high-accuracy 3D shape measurement with saturated fringe patterns. By allowing the use of saturated patterns, this finding overcomes one of the problems associated with the limited dynamic range of cameras. Both simulation and experimental data demonstrated that by properly using an $\mathrm{N}$-step phase-shifting algorithm, high-contrast surfaces can be properly measured with a single exposure even though some areas are saturated. This finding leads to possible general solution to a long-standing problem in the optical metrology community: optically measure shiny metal parts or high-contrast surfaces using a conventional digital fringe projection (DFP) technique. 


\section{Acknowledgments}

This study was sponsored by the National Science Foundation (NSF) under grant numbers CMMI-1523048. The views expressed in this paper are those of the authors and not necessarily those of the NSF.

\section{References}

[1] S. Zhang and S.-T. Yau, "High dynamic range scanning technique," Opt. Eng. 48(3), 033,604 (2009).

[2] C. Waddington and J. Kofman, "Analysis of measurement sensitivity to illuminance and fringe-pattern gray levels for fringe-pattern projection adaptive to ambient lighting,” Opt. Laser Eng. 48, 251-256 (2010).

[3] H. Jiang, H. Zhao, and X. Li, "High dynamic range fringe acquisition: A novel 3d scanning technique for high-reflective surfaces," Opt. Laser Eng. 50, 1484-1493 (2012).

[4] H. Zhao, X. Liang, X. Diao, and H. Jiang, "Rapid in-situ 3d measurement of shiny object based on fast and high dynamic range digital fringe projector," Opt. Laser Eng. 54, 170-174 (2014).

[5] L. Ekstrand and S. Zhang, "Auto-exposure for three-dimensional shape measurement with a digital-light-processing projector," Opt. Eng. 50(12), 123,603 (2011).

[6] L. Ekstrand and S. Zhang, "Automated high-dynamic range three-dimensional optical metrology technique," in ASME 2014 International Manufacturing Science and Engineering Conference (Detroit, Michigan, 2014).

[7] D. Li and J. Kofman, "Adaptive fringe-pattern projection for image saturation avoidance in 3D surface-shape measurement," Opt. Express 22, 9887-9901 (2014).

[8] C. Waddington and J. Kofman, "Camera-independent saturation avoidance in measuring high-reflectivity-variation surfaces using pixel-wise composed images from projected patterns of different maximum gray level," Opt. Comm. 333, 32-37 (2014).

[9] C. Waddington and J. Kofman, "Modified sinusoidal fringe-pattern projection for variable illuminance in phase-shifting 3-D surface-shape metrology," Opt. Eng. 53, 084,109 (2014).

[10] J. Jeong and M. Kim, "Adaptive imaging system with spatial light modulator for robust shape measurement of partially specular objects," Opt. Express 18, 27,787-27,801 (2010).

[11] Y. Yamaguchi, H. Miyake, O. Nishikawa, and T. Iyoda, "Shape Measurement of Glossy Objects by Range Finder with Polarization Optical System," Gazo Denshi Gakkai Kenkyukai Koen Yoko (in Japanese) 200, 43-50 (2003). 
[12] B. Salahieh, Z. Chen, J. J. Rodriguez, and R. Liang, "Multi-polarization fringe projection imaging for high dynamic range objects," Opt. Express 22, 10,064-10,071 (2014).

[13] Q. Hu, K. G. Harding, X. Du, and D. Hamilton, "Shiny Parts Measurement using Color Separation," in Proc. SPIE, vol. 6000, pp. 6000D1-8 (2005).

[14] S. Zhang, "Flexible 3D shape measurement using projector defocusing: Extended measurement range," Opt. Lett. 35(7), 931-933 (2010).

[15] Y. Li, C. Zhao, Y. Qian, H. Wang, and H. Jin, "High-speed and dense threedimensional surface acquisition using defocused binary patterns for spatially isolated objects," Opt. Express 18(21), 21,628-21,635 (2010).

[16] S. Zhang, "Composite phase-shifting algorithm for absolute phase measurement," Opt. Laser Eng. 50(11), 1538-1541 (2012).

[17] Z. Li, K. Zhong, Y. Li, X. Zhou, and Y. Shi, "Multiview phase shifting: a full-resolution and high-speed 3D measurement framework for arbitrary shape dynamic objects," Opt. Lett. 38(9), 1389-1391 (2013).

[18] K. Zhong, Z. Li, Y. Shi, C. Wang, and Y. Lei, "Fast phase measurement profilometry for arbitrary shape objects without phase unwrapping," Opt. Laser Eng. 51(11), 12131222 (2013).

[19] W. Lohry and S. Zhang, "High-speed absolute three-dimensional shape measurement using three binary dithered patterns," Opt. Express 22(22), 26,752-26,762 (2014).

[20] W. Lohry, V. Chen, and S. Zhang, "Absolute three-dimensional shape measurement using coded fringe patterns without phase unwrapping or projector calibration," Opt. Express 22(2), 1287-1301 (2014).

[21] Y. Xu, L. Ekstrand, J. Dai, and S. Zhang, "Phase error compensation for threedimensional shape measurement with projector defocusing," Appl. Opt. 50(17), 25722581 (2011).

[22] Y. Chen, Y. He, and E. Hu, "Phase deviation analysis and phase retrieval for partial intensity saturation in phase-shifting projected fringe profilometry," Opt. Comm. 281, 3087-3090 (2008).

[23] E. Hu, Y. He, and W. Wu, "Further study of the phase-recovering algorithm for saturated fringe patterns with a larger saturation coefficient in the projection grating phase-shifting profilometry," Optik 121, 1290-1294 (2010).

[24] C. Jiang, T. Bell, and S. Zhang, "High dynamic range real-time 3D shape measurement," Opt. Express 24(7), 7337-7346 (2016). 